\title{
Workplace-related rehabilitation after foot fractures
}

\author{
Eckehard Froese \\ Department of Rehabilitation. Social Accident Insurance (VBG), Germany. eckehard.froese@vbg.de
}

Background: For injured workers facing increased physical requirements the VBG, a German Social Accident Insurance Institution, provides 2 types of outpatient medical treatment for the last phase of rehabilitation. One option is a functionally orientated extended physiotherapy including the opportunity for work-ability testing at the workplace with a gradual increase of workload and working time. Second option is a newly-developed multidisciplinary workplace-related rehabilitation integrating the specific work-relevant functional flows in therapy under the direction of a qualified physician and therapists in rehab facilities.

Objective: To evaluate the effects of workplace-related rehabilitation after foot fractures in outpatient rehab facilities. Methods: Data of all completed cases with severe fractures of the calcaneus or the ankle joint, undergoing workplace-related therapy in $2013(\mathrm{n}=27)$, were compared to consecutive data of 27 completed cases undergoing extended physiotherapy.

Findings: Mean age in both groups was 48 years. In the extended physiotherapy group 14 patients additionally received a work-ability testing directly at the workplace. Mean duration from accident to capacity for work was 169.81 days (SD 60.4) for extended physiotherapy and 176.81 days (SD 49.3) for workplace-related rehabilitation. 23 patients with extended physiotherapy and 26 patients with workplace-related rehabilitation maintained work ability 3 months after achieving full capacity for work. Subsequent rehab measures after regaining capacity for work were needed in 6 cases in the extended physiotherapy group and in one case in the workplace-related rehabilitation group.

Discussion: Though no statistically verifiable difference in duration of incapacity for work between both groups was found, the results provide apparently evidence that multidisciplinary workplace-related rehabilitation is more sustainable. It is suggested, that a systematic approach and individually tailored multidisciplinary training of the specific work-relevant movement patterns under constant medical and therapeutic direction lead to more stable rehabilitation results.

Conclusion: Further research is needed to consolidate our empirical findings. 